\title{
Educando la competencia social en Perú. Programa de prevención del maltrato escolar
}

\section{Teaching Social Competence in Peru. A Bullying Prevention Program}

\author{
María Nery Sampén-Díaz (1) msampen@usat.edu.pe \\ María Carmen Aguilar-Ramos (2) maguilar@uma.es \\ Juan Carlos Tójar-Hurtado (2) jctojar@uma.es \\ (1) Universidad Católica Santo Toribio de Mogrovejo \\ (2) Universidad de Málaga \\ (Recibido: 28 de noviembre de 2014; Aceptado para su publicación: 11 de noviembre de 2015)
}

Cómo citar: Sampén-Díaz, M. N., Aguilar-Ramos, M. C. y Tójar-Hurtado, J. C. (2017). Educando la competencia social en Perú. Programa de prevención del maltrato social. Revista Electrónica de Investigación Educativa, 19(1), 46-57. Recuperado de https://doi.org/10.24320/redie.2017.19.1.1013

\section{Resumen}

La presente investigación sobre maltrato escolar realizada en Perú, aplicando un enfoque de investigación mixto (cualitativo-cuantitativo), evalúa un programa de prevención basado en la competencia social e interpersonal. El procedimiento incluye un diseño cuasi-experimental que se desarrolló en dos fases: 1) análisis y detección de la situación de maltrato y 2) diseño, aplicación y evaluación del programa. Participaron 176 estudiantes de tres instituciones educativas de Chiclayo, Perú. Las técnicas de recogida de datos fueron cuestionarios, observación participante y fichas de evaluación. Los resultados indican que el programa contribuyó a potenciar aprendizajes enmarcados dentro de la competencia social. Tras la aplicación del programa, los estudiantes mejoraron en actitudes de convivencia con sus iguales, diálogo, expresión de sentimientos, comprensión y empatía con quien sufre, así como en amistad y solidaridad en situaciones de maltrato escolar, lo que pone de manifiesto la importancia de potenciar el aprendizaje de la competencia social.

Palabras clave: Prevención, maltrato infantil, evaluación de programas, bullying, competencia.

\section{Abstract}

This study on bullying, carried out in Peru, draws on a mixed research approach (qualitative and quantitative) to assess a prevention program based on social and interpersonal competence. The procedure includes a quasi-experimental design developed in two phases: 1) the analysis and detection of bullying situations, and 2) the design, implementation and assessment of the program. The study included 176 students from three educational institutions in Chiclayo, Peru. 
Data was collected through questionnaires, participant observation and assessment sheets. The results show that the program boosted social competence skills. After implementation of the program, students' attitudes improved, showing better coexistence with peers, dialog, expression of feelings, understanding and empathy towards those who suffer, as well as friendship and solidarity in bullying situations. This highlights the importance of strengthening social competence skills.

Keywords: Prevention, child abuse, program evaluation, bullying, interpersonal competence.

\section{Introducción}

El aprendizaje de la convivencia es uno de los mayores retos que deberá afrontar la educación del siglo XXI. La formación integral del estudiante, como ser individual y social, no sólo abarca la adquisición de conocimientos científicos y tecnológicos, sino que, además, requiere potenciar y desarrollar habilidades, actitudes y valores que le permitan aprender y convivir con sus iguales. Por ello, es necesario reflexionar y desarrollar una pedagogía de la convivencia, orientada a desarrollar en los estudiantes aprendizajes ineludibles como: solidaridad, diálogo, cooperación, tolerancia, habilidades sociales, gestión de conflictos interpersonales, etc. Aprendizajes que a los estudiantes les permitan una mejor interacción entre sus pares; disfrutar de una convivencia no exenta de conflictos y problemas y, al mismo tiempo, de relaciones interpersonales que contribuyan a generar un clima positivo, en donde la resolución de los problemas favorezca un desarrollo personal y social (Martin, del Barrio y Echeita, 2003).

Estudios referentes a la convivencia escolar enfatizan que la educación es un proceso caracterizado por la relación interpersonal impregnada de fenómenos socio-afectivosemocionales, estando presente la dimensión social en el proceso de aprendizaje individual y autónomo (Trianes y García, 2002). De lo expuesto hasta ahora se desprende la urgente necesidad de intensificar la acción educativa como condición necesaria para lograr una auténtica convivencia basada en valores (Frontado, 2003) y, además, que esta educación en valores, ha de constituir un elemento de peso en el currículo de las diversas etapas educativas (Ortega y Del Rey, 2007).

Por su parte, los estudios sobre maltrato escolar (Chaux, 2012; Díaz, 2007; Fernández, 2003; Levine y Tamburrino, 2014; Mendoza, 2012; Smith y Low, 2013) coinciden en señalar sus efectos nocivos en el desarrollo personal, social, académico, emocional y moral de niños y adolescentes que participan en la dinámica del maltrato escolar. Impresiona y preocupa conocer las implicaciones que genera el participar, ya sea como agresor, víctima u observador en situaciones de maltrato.

La revisión ya clásica de estudios científicos desde diferentes perspectivas (biológicas, psicológicas y socioculturales) ofrece el trabajo pionero, realizado en Noruega por Olweus (1993), quien mostraba cómo la violencia entre escolares afectaba directamente al $15 \%$ de los estudiantes entre 7 y 16 años, enfatizando -además- que era una conducta con mayor incidencia en el género masculino.

En España, el estudio nacional de mayor relevancia sobre este fenómeno entre iguales, en la enseñanza secundaria obligatoria, fue realizado por primera vez en 1999 (Defensor del Pueblo, 2000) y posteriormente en el 2006 (Defensor del Pueblo, 2007). El último informe mostró que el $3.9 \%$ sufría agresiones físicas directas, el $6.4 \%$ amenazas para provocar miedo y, en la categoría exclusión social, el $10.5 \%$ declaraba ser ignorado. En relación a las agresiones verbales, el $26.7 \%$ expresaba haberlas sufrido, el $27.1 \%$ concretamente por medio de insultos; el $31.6 \%$, en la modalidad más frecuente de agresión, hablar mal de ellos, y el 5.5\% víctimas de abusos por medio de las nuevas Tecnologías de Información y Comunicación (TIC). Los resultados en función del género evidencian que los estudiantes de género masculino tienden a tener 
comportamientos más maltratadores que los de género femenino, y la sala de clase y el patio son los escenarios donde se producen más los maltratos.

En el contexto latinoamericano, el estudio nacional de violencia escolar realizado por el Ministerio del Interior de Chile (Valdivieso, 2009), muestra que los estudiantes y docentes manifiestan que la frecuencia de agresiones es alta, en un $34 \%$ y $51.2 \%$, respectivamente, con mayor incidencia en los estudiantes de colegios públicos. El tipo de violencia que predomina es la agresión psicológica, según la percepción del $96 \%$ de estudiantes y docentes; seguido de las agresiones físicas, el $83.3 \%$ y el $61.1 \%$, respectivamente. El $73.9 \%$ del total considera que se producen por rivalidad o presión del grupo.

En México, el estudio denominado "Disciplina, violencia y consumo de sustancias nocivas a la salud en escuelas de educación primaria y secundaria", realizado por el Instituto Nacional para la Evaluación de la Educación (INEE) (Aguilera, Muñoz y Orozco, 2007), mostró que el 43.6\% de los estudiantes había sido víctima de robos dentro de la propia escuela, y sólo el 1.3\% declaraba haber participado en estas situaciones; el 14.1\% había sido lastimado físicamente, el 13.6\% había sufrido burlas y el $13.1 \%$ había recibido amenazas; en general, el $18.2 \%$ de estudiantes de educación secundaria había sufrido violencia.

En Perú, la Comisión Nacional para el Desarrollo y Vida sin Drogas (2007) realizó una investigación en la que participaron 65,041 estudiantes de 516 colegios (416 públicos y 100 privados), los resultados mostraron que el $15.3 \%$ había sido víctima de una o más agresiones físicas en su vida escolar. El 20.2\% reconocía que el agresor o agresores habían sido sus propios compañeros de clase.

Oliveros y Barrientos (2007) desarrollaron una investigación con 185 estudiantes de cuarto grado de educación primaria y quinto grado de educación secundaria de un colegio particular de Lima. Los resultados revelaron que el 54.7\% había sufrido bullying; el 38.7\% intimidación verbal (el maltrato de mayor incidencia) siendo los apodos o sobrenombres el tipo más frecuente de agresión. El 84.3\% no defendió a sus compañeros.

Oliveros et al. (2009) estudiaron la frecuencia de la intimidación en colegios nacionales, en los que había existido violencia política, para así determinar los factores de riesgo asociados. Participaron 1,633 estudiantes de educación secundaria, de cinco departamentos del país: Ayacucho (Huamanga), Cusco (Sicuani), Junín (Satipo), Huancavelica y Lima (Ñaña). Los resultados evidenciaron un promedio de $50.7 \%$ de intimidación y las variables asociadas: los apodos, golpes, falta de comunicación, llamar homosexual, resaltar defectos físicos, escupir, obligar a hacer cosas que no se deseaban, discriminación e insulto por correo electrónico. Los autores concluyeron que la intimidación tiene origen multicausal, ocasiona problemas en la salud y fobia escolar; el pronóstico a largo plazo para víctimas y agresores es negativo, al grado en que los agresores pueden tener problemas con la ley en el futuro.

Desde diferentes ámbitos, la evidencia empírica pone de relieve la necesidad de educar la competencia social. En los últimos años se ha evidenciado el deterioro de la convivencia escolar, manifestada en la intolerancia a la diversidad cultural, religiosa, étnica, social y económica, entre otras, y han aumentado las conductas de riesgo en niños y adolescentes, lo que ha ocasionado un interés generalizado por la educación para la convivencia y la recuperación de la función moral de la educación, la insistencia en el desarrollo de habilidades sociales, de la empatía, el altruismo, la educación cívica y el aprendizaje de la solidaridad, ya que al igual que se aprende la solidaridad, la honradez o el respeto a la diferencia, se puede aprender también la violencia y la intolerancia (Torrego y Moreno, 2003). 
En este contexto, hemos considerado imprescindible diseñar una intervención para favorecer el aprendizaje y desarrollo de la competencia social en los adolescentes, para hacer posible el bienestar personal y social en las aulas y para potenciar el desarrollo del estudiante en sus diferentes dimensiones: intelectual o cognitiva, socio-afectiva, ética y moral. Estas motivaciones fundamentan el contenido del programa "Desarrollemos la competencia social para convivir en armonía con los demás", cuyos fundamentos se apoyan en la antropología filosófica que explica la dimensión trascendente de la persona y contempla la singularidad del ser humano para hacerle consciente de sí mismo, como un acto creativo de su ser único e irrepetible, donde es importante el conocimiento de la verdad, el bien y la belleza, pero teniendo en cuenta que su existencia se desarrolla en interacción con los demás, puesto que el ser humano necesita el encuentro con el otro para ser escuchado, animado y poder comunicarse (Arismendi, 1992; García, 1981; Gevaert 1987; Gonzales y Negreiros, 2001; Yepes, 2003; Killam, Roland y Weber, 2014).

El modelo pedagógico que ha guiado el desarrollo del programa ha sido el constructivista, permitiendo que los estudiantes fueran los protagonistas de sus aprendizajes, a través de una metodología participativa, en la que han realizado análisis de casos, dramatizaciones, análisis de imágenes, dialogo, trabajo individual y grupal, dilemas morales, expresión de sentimientos y puntos de vista, expresión de opiniones, formulación de críticas, preguntas y respuestas para animar o reforzar los aprendizajes, para ayudar a comprender un contenido, para facilitar su participación, para saber el grado de asimilación y comprensión de la tarea (Johnson, Kieling y Cooper, 2013). En consecuencia, el estudiante ha desempeñado un rol dinámico, activo, eficaz y constructivo dentro del proceso de enseñanza y aprendizaje, y el docente ha sido el mediador, guía, facilitador y dinamizador de los procesos motivacionales, cognitivos y actitudinales. Se ha puesto énfasis en los cambios que se deben operar en el proceso de enseñanza-aprendizaje, a fin de lograr la más amplia interacción entre el docente y los alumnos y entre alumnos-alumnos, favoreciendo el aprendizaje cooperativo y el desarrollo de habilidades cognitivas, sociales y el desarrollo moral, aspectos que contribuyen a mejorar la dinámica y el clima del aula.

Se ha considerado que la formación de la competencia social en los estudiantes, de todos los niveles educativos, representa una necesidad y un reto para esta sociedad globalizada, diversa y multicultural; requiere desarrollar capacidades, conocimientos y actitudes para aprender a vivir y convivir con los demás, gestionando y manteniendo relaciones interpersonales positivas y constructivas con personas de diferentes culturas, y exige que los docentes, directivos, padres de familia y estudiantes reflexionen y tomen conciencia de la magnitud de los daños que genera el maltrato escolar, en sus diferentes manifestaciones, y se pregunten qué se está haciendo desde los espacios educativos para prevenir y atender estas conductas.

Las reflexiones sobre la necesidad de favorecer el aprendizaje de la competencia social y la toma de conciencia del papel de la educación para favorecer la convivencia en el ámbito escolar han justificado la aplicación del programa de educación de la competencia social a los estudiantes de segundo grado de educación secundaria con el fin de prevenir el maltrato escolar. El programa ha contemplado de manera integral y equilibrada el desarrollo de conocimientos, habilidades, valores y actitudes, donde van a influir el contexto, los recursos y el enfoque pedagógico desarrollado en el aula por el docente (De Zubirías, 2006; Altarejos, 1986). Se desarrolló en 15 sesiones de aprendizaje, de 2 horas pedagógicas (90 minutos) semanales, en las que se trató de profundizar en la temática más solicitada por los estudiantes.

\section{Método}

En el estudio participaron 176 estudiantes de segundo grado de educación secundaria de tres instituciones educativas estatales: masculina, femenina y mixta de la ciudad de Chiclayo (Perú), pertenecientes a zonas urbanas marginales, las edades de los participantes fluctúan entre 12 y 16 años, el 80\% tiene 13 años, el 11\% tiene 14 años y un 6\% cuenta con 16 años. Se contó con el consentimiento informado de los padres de familia para que de manera voluntaria los 
estudiantes respondieran a los cuestionarios y participaran en el programa, orientado al aprendizaje de la competencia social para prevenir conductas de maltrato escolar. La muestra obtenida a través de un muestreo teórico (Tójar, 2006) ha representado adecuadamente el fenómeno de estudio sobre el que se deseaba experimentar el programa.

El marco metodológico del estudio fue mixto (cuantitativo-cualitativo). El fenómeno del maltrato escolar, complejo y diverso, es preciso abordarlo desde una perspectiva amplia y profunda. Requiere una visión interdisciplinar y actuar desde planteamientos teóricos y metodológicos apropiados (Hernández, Fernández y Baptista, 2006). Este enfoque ha permitido abarcar la exploración e identificación de los distintos problemas, que requerían atención pedagógica, la evaluación de fortalezas y las acciones para la mejora del programa de educación de la competencia social. En este sentido, se ha utilizado un diseño mixto de tipo anidado o incrustado concurrente de modelo dominante.

En la primera fase de la investigación se aplicó un cuestionario pre-test con preguntas cerradas, para detectar el maltrato en los diferentes roles (agresor, víctima y observador) y 9 dimensiones de maltrato escolar (ver tabla I), y preguntas abiertas, para descubrir el contexto familiar y las necesidades de aprendizaje de los estudiantes.

Tabla I. Presencia de agresiones físicas directas

\begin{tabular}{|c|c|c|}
\hline Dimensión & Indicadores & Roles sociales \\
\hline $\begin{array}{l}\text { Agresiones físicas } \\
\text { directas }\end{array}$ & $\begin{array}{l}\text { Puñetes, patadas, empujones, pellizcos, golpes, } \\
\text { quemaduras, peleas con armas (cuchillos, pistola, } \\
\text { chavetas, navajas). }\end{array}$ & $\begin{array}{l}\text { Agresor } \\
\text { Víctima } \\
\text { Observador }\end{array}$ \\
\hline $\begin{array}{l}\text { Agresiones físicas } \\
\text { indirectas }\end{array}$ & $\begin{array}{l}\text { Pequeños hurtos, destrozo de pertenencias, esconder } \\
\text { objetos, provocaciones. }\end{array}$ & $\begin{array}{l}\text { Agresor } \\
\text { Víctima } \\
\text { Observador }\end{array}$ \\
\hline $\begin{array}{l}\text { Agresiones } \\
\text { verbales: directas }\end{array}$ & $\begin{array}{l}\text { Insultos, ofender poniendo en evidencia características } \\
\text { distintivas de la víctima, menospreciar en público, poner } \\
\text { apodos. }\end{array}$ & $\begin{array}{l}\text { Agresor } \\
\text { Víctima } \\
\text { Observador }\end{array}$ \\
\hline $\begin{array}{l}\text { Agresiones } \\
\text { verbales indirectas }\end{array}$ & $\begin{array}{l}\text { Hablar mal del compañero, propagar rumores y mentiras; } \\
\text { utilizar el teléfono celular, correo electrónico, Facebook } \\
\text { o Twitter para acciones de difamación, amenazas y burlas. }\end{array}$ & $\begin{array}{l}\text { Agresor } \\
\text { Víctima } \\
\text { Observador }\end{array}$ \\
\hline $\begin{array}{l}\text { Intimidaciones, } \\
\text { chantaje y } \\
\text { amenazas }\end{array}$ & $\begin{array}{l}\text { Provocar miedo, obtener algún objeto o dinero, obligar a } \\
\text { hacer cosas que no quiera hacer, amenazar, utilizar armas } \\
\text { (cuchillos, pistola, chavetas, navajas). }\end{array}$ & $\begin{array}{l}\text { Agresor } \\
\text { Víctima } \\
\text { Observador }\end{array}$ \\
\hline $\begin{array}{l}\text { Aislamiento y } \\
\text { exclusión social }\end{array}$ & $\begin{array}{l}\text { Impedir la participación de la víctima, aislarle del grupo, } \\
\text { ignorar su presencia, no contar con él o ella para } \\
\text { actividades del grupo. }\end{array}$ & $\begin{array}{l}\text { Agresor } \\
\text { Víctima } \\
\text { Observador }\end{array}$ \\
\hline Acoso racial & $\begin{array}{l}\text { Poner apodos (motes) racistas. } \\
\text { Expresar frases estereotipadas despectivas. }\end{array}$ & $\begin{array}{l}\text { Agresor } \\
\text { Víctima } \\
\text { Observador }\end{array}$ \\
\hline Contexto familiar & $\begin{array}{l}\text { Maltratos en el contexto intrafamiliar. } \\
\text { Apoyo parental. }\end{array}$ & $\begin{array}{l}\text { Agresor } \\
\text { Víctima } \\
\text { Observador } \\
\text { Control parental } \\
\text { indirecto }\end{array}$ \\
\hline $\begin{array}{l}\text { Relaciones } \\
\text { interpersonales } \\
\text { entre integrantes } \\
\text { de la IE y de su } \\
\text { familia. }\end{array}$ & $\begin{array}{l}\text { Relación interpersonal entre estudiantes, estudiantes y } \\
\text { profesores, estudiantes y adultos, profesores y padres de } \\
\text { familia. }\end{array}$ & \\
\hline
\end{tabular}

Este análisis y detección de necesidades puso en evidencia la situación de maltrato escolar, el contexto familiar y las necesidades de aprendizaje. Asimismo, se concretaron los Temas Considerados Importantes por los Estudiantes (TICE) centrados en: conflictos en el aula; conducta 
agresiva; escasa práctica de valores: amistad, solidaridad, respeto y cooperación, discriminación, baja autoestima, escasa atención por parte del docente a los insultos de los compañeros, trato deficiente de los profesores a los estudiantes y violencia familiar. En la segunda fase, a partir de la información recogida en el diagnóstico inicial y en los TICE, se diseñó y elaboró el programa de prevención del maltrato escolar, denominado "Desarrollemos la competencia social para convivir en armonía con los demás", que se aplicó con el fin de educar la competencia social. Las sesiones de dicho programa se desarrollaron en los tres contextos educativos y significaron una reflexión permanente, sobre la forma en que se establecían las relaciones interpersonales en los grupos de trabajo, la forma en que respondían a las tareas asignadas, la organización, el liderazgo del coordinador de grupo, las manifestaciones de respeto entre los iguales y la tolerancia a las diferencias. Dichas reflexiones se registraron en un diario de campo, recogiendo así las incidencias durante la permanencia en dichas instituciones educativas. Además, significó un instrumento más para el análisis de la pertinencia de las estrategias utilizadas en las sesiones. Durante el desarrollo del programa, tras cada sesión, se recogió información a través de fichas de evaluación para conocer el grado de satisfacción de los estudiantes, en la participación en los talleres, la pertinencia de los temas, los aprendizajes desarrollados, las fortalezas de la temática abordada y las acciones por mejorar durante el proceso de enseñanza y aprendizaje. Al finalizar, se evaluó el programa con el propósito de valorar sus efectos y se aplicó el cuestionario post-test.

El análisis de la información recogida por medio de las técnicas e instrumentos cuantitativos (cuestionario pre-post-test) como cualitativos (cuestionarios de preguntas abiertas, observación participante, fichas de evaluación) se realizó aplicando los programas informáticos SSPS y Excel, y las pruebas estadísticas aplicadas fueron la prueba de independencia Chi cuadrada y la prueba Z de comparación de proporciones. Para el análisis de contenido se empleó el programa Atlas.ti, iniciando este análisis con la reducción cualitativa de datos, priorizando el criterio de la relevancia interpretativa y eliminando lo superfluo o redundante, tratando en lo posible de no perder la esencia de lo expresado por los estudiantes (Tójar, 2006).

\section{Resultados}

De acuerdo con los objetivos de la investigación se presentan los resultados, que consideramos más relevantes, relacionados con las variables: maltrato escolar y necesidades e intereses de aprendizajes de los estudiantes, el sistema ecológico (que incide en la dinámica del maltrato) y la competencia social.

Los datos de maltrato escolar, en sus diferentes manifestaciones, muestran que están presentes en las instituciones educativas, tanto de las zonas urbanas como marginales. Destacamos que el porcentaje más alto de maltrato escolar se produce en la institución educativa masculina: 28\% y $42 \%$, en los grupos Control y Experimental, respectivamente, y representa la dimensión de agresiones físicas directas rol agresor (ver figura 1) y las indirectas 32\% y 33\%, en los grupos Control y Experimental, respectivamente. 


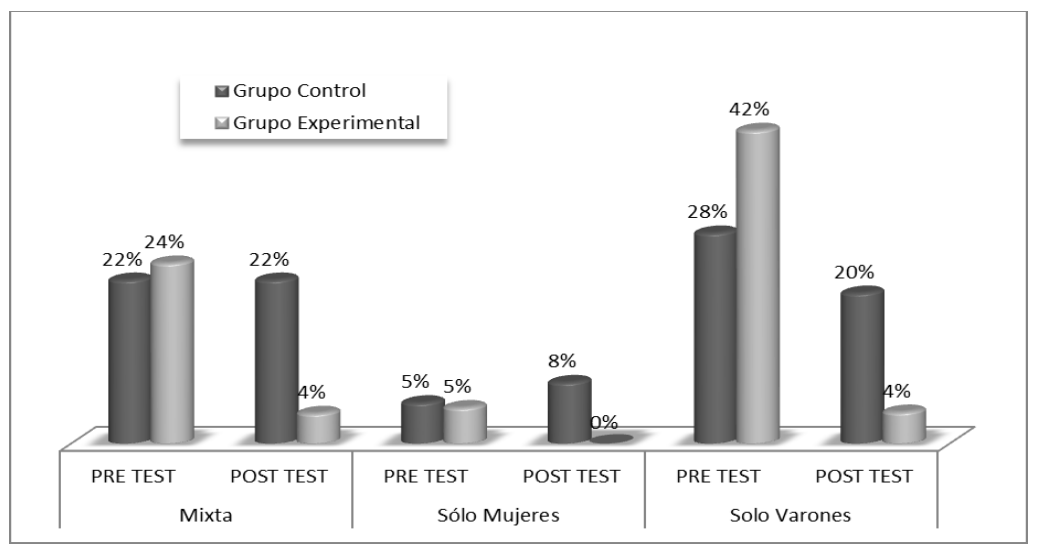

Figura 1. Presencia de agresiones físicas directas

En la institución educativa femenina, los grupos Control y Experimental, respectivamente, presentan el $62 \%$ y $48 \%$, en la dimensión agresiones verbales indirectas rol víctima (ver figura 2) hablar mal del compañero, propagar rumores y mentiras, utilizar el teléfono celular, correo electrónico, Facebook, eBuddy, Twitter, para acciones de difamación, amenazas y burlas.

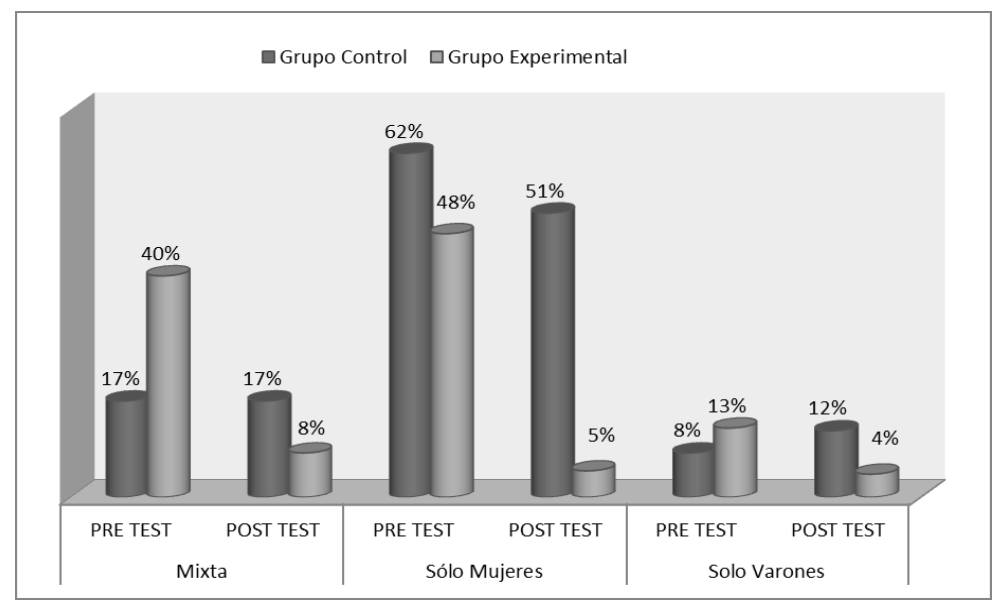

Figura 2. Presencia de víctimas de agresiones verbales indirectas

Los datos cualitativos sobre las necesidades de aprendizaje para convivir y aprender en espacios educativos saludables, se categorizaron como TICE y requerían de orientación y atención pedagógica. Estos resultados revelaron las conductas de maltrato escolar más comunes en las tres instituciones educativas: conflictos en el aula; conducta agresiva; escasa práctica de valores: amistad, solidaridad, respeto y cooperación; discriminación; baja autoestima; escasa atención por parte del docente a los insultos de los compañeros, el trato deficiente de los profesores a los estudiantes y violencia familiar.

Respecto a las características del sistema ecológico que inciden en la dinámica del maltrato escolar, se aprecia en los datos que se trata de un fenómeno multicausal, en el que tienen influencias decisivas los diferentes contextos donde se desarrollan e interactúan los estudiantes. En el microsistema familiar, lugar privilegiado para aprender a convivir con los demás, la mayoría de los estudiantes de la institución educativa masculina pertenece a familias disfuncionales, y al relacionar este dato con los datos cuantitativos se ha observado que el $29 \%$ perciben agresiones en el contexto familiar, característica considerada como factor de riesgo en la probabilidad de 
asumir conductas maltratadoras. En cuanto a los estudiantes de la institución educativa mixta y femenina, el alto porcentaje que vive en compañía de sus progenitores mantiene una buena relación familiar. En este contexto, consideramos relevante destacar que aproximadamente la mitad de los escolares implicados en este estudio manifiesta que sus padres no dedican tiempo para conversar con ellos, no conocen sus actividades, carecen de reglas claras en la casa, así como de actividades conjuntas.

En relación al microsistema escolar, contexto donde los estudiantes interactúan con sus iguales, las relaciones interpersonales entre pares y entre estudiantes y profesores presentan conductas de malos tratos, lo que nos lleva a concluir que los contextos más cercanos, a los estudiantes, necesitan vitalizarse con estrategias educativas que orienten un aprendizaje de la convivencia.

En lo que se refiere al mesosistema, en el que se considera el conjunto de microsistemas que interactúan entre sí, se aprecia la necesidad de una integración entre familia y escuela, utilizando estrategias adecuadas y orientadas a prevenir o disminuir el maltrato entre pares. En la medida en que los estudiantes perciban la existencia de una estrecha comunicación y mejor entendimiento entre docentes y padres de familia su formación será más integral.

Respecto la estructura, objetivos, temática, estrategias, recursos y evaluación, del programa "Desarrollemos la competencia social para convivir en armonía con los demás", durante su desarrollo los estudiantes estuvieron muy motivados, entusiastas, poniéndolo de manifiesto con su participación activa y permanente, siendo los protagonistas de sus aprendizajes. En cuanto a su efecto fue positivo, se comprobó estadísticamente, con un nivel de confianza de al menos el 95\% (alfa inferior al 5\%) las hipótesis de disminución de las proporciones de presencia del maltrato escolar en las tres instituciones educativas, en los diferentes roles (agresor, víctima y observador) y en las 9 dimensiones de maltrato escolar consideradas en la investigación.

En relación con la educación de la competencia social, tras la aplicación del programa, hemos podido evaluar comparativamente, en el pre-post-test, los logros alcanzados en la disminución del maltrato escolar. Los resultados en el post-test mostraron que la temática desarrollada contribuyó a interiorizar los valores eje para una adecuada convivencia escolar, observando una mejora cualitativa en sus actitudes.

Los estudiantes mostraron una mayor capacidad empática, la utilización de un estilo de comunicación asertiva, una mejor actitud de escucha, capacidad de tolerancia y respeto a otras ideas, formas culturales y una mejor aceptación de sus iguales en los grupos de trabajo. Asimismo, desarrollaron la habilidad para participar de manera constructiva y pacífica en la resolución de conflictos, utilizaron estrategias más humanas de resolución de conflictos, como el diálogo, el saber escuchar, el saber esperar turno y construir consensos en las discrepancias, y que se aprecia en los resultados, el avance muy significativo en el uso de herramientas: diálogo, consenso y mediación. También asumieron que los conflictos representan oportunidades para crecer en la comprensión y solución de problemas. Desde la perspectiva de las habilidades cognitivas, el programa aplicado contribuyó al desarrollo de capacidades de reconocimiento y aprendizaje, en relación con que un problema tiene causalidad múltiple y que varias alternativas de solución pueden ser formuladas, lo que favorece la toma de conciencia en la interacción con pares y familia.

\section{Discusión}

El porcentaje más alto de maltrato escolar en la dimensión agresiones físicas directas e indirectas, en el rol agresor, hallado en la institución educativa masculina, coincide con la investigación realizada por Piñero (2010) quien encontró que los niveles de implicación en violencia son superiores en chicos que en chicas, y que la violencia física y las amenazas son empleadas con mayor frecuencia en chicos que en chicas. Olweus (1993) reafirma que los malos tratos escolares 
son una conducta con mayor incidencia en el género masculino. Asimismo, el porcentaje más alto de víctimas en la dimensión agresiones físicas indirectas, como acciones de difamación, amenazas y burlas, etc., que se ha encontrado en la institución educativa femenina coindice con el estudio de Del Barrio, Barrios, Meulen y Gutiérrez (2003) que revela que estas formas indirectas de maltrato es de mayor incidencia en las chicas.

La mirada ecológica de los contextos familia y escuela en los que interactúan los estudiantes nos remite a Tuvilla (2004), quien considera que toda acción preventiva debe organizarse en una perspectiva ecológica, que se inicie en la familia, continúe en los contextos educativos y esté apoyada por acciones en el ámbito socio-comunitario. Este pensamiento refuerza el enfoque que hemos pretendido desarrollar en el análisis, interpretación e intervención del fenómeno del maltrato escolar en estos escenarios educativos. Desde esta perspectiva se aprecia la necesidad de una integración entre familia y escuela, de forma que se puedan utilizar las estrategias adecuadas orientadas a prevenir o disminuir el maltrato entre pares.

Respecto a los estudiantes que viven en familias disfuncionales y manifiestan la falta de atención por parte de sus padres, los resultados concuerdan con el estudio con escolares chilenos de Santander et al. (2008), quienes consideraron la influencia de la familia como factor protector de conductas de riesgo, ya que el $66.5 \%$ percibe que sus familias son disfuncionales, y con Chaux (2012) en donde se encontró, referente a la perpetración de violencia, que aquellos jóvenes que fueron maltratados físicamente en su niñez tenían un riesgo sustancialmente más alto de involucrarse, durante la adolescencia, en delincuencia, peleas físicas, intimidación escolar y violencia en la relación de pareja.

El programa para aprender la competencia social ha contribuido a desarrollar capacidades para reconocer y aprender que un problema tiene varias causas y se pueden formular varias alternativas de solución, lo que conlleva a ser consciente de sus actos en su interacción con sus pares y familiares. Al respecto, Segura (2007) considera esta capacidad cognitiva como la capacidad que hace posible el amor y, por tanto, nos hace seres humanos. Enfatiza, además, que las personas agresivas, especialmente las de comportamiento más violento suelen carecer de esta capacidad y, por lo tanto, consideran como la violencia como único recurso de resolución de conflictos.

En cuanto a la habilidad para participar de manera constructiva y pacífica en la resolución de conflictos, asumiendo que los conflictos representan oportunidades para crecer en la comprensión y solución de problemas, lo relacionamos con Hazas (2010), quien señala que la presencia de conflictos ayuda a aprender nuevas y mejores maneras de responder a los problemas y funciona como motor de cambio, personal y social.

Con relación a las actitudes de respeto, tolerancia, solidaridad, cooperación, amistad, escucha activa, etc., los estudiantes han mostrado una toma de conciencia cada vez más viva de su propia responsabilidad, lo que conlleva mejorar el clima en el aula. Galeano (2005) considera la solidaridad como valor social por excelencia y muy necesario para la convivencia. Asimismo, Segura (2004) plantea en la educación en valores la necesidad de desarrollar el diálogo, la solidaridad, el aprender a escuchar, el saber hacer una crítica constructiva de la realidad personal y social y el ponerse de acuerdo sobre las normas de convivencia.

\section{Conclusiones}

La aplicación del programa para la educación de la competencia social ha contribuido a incrementar la capacidad empática y las actitudes asertivas poniendo en evidencia una mejora significativa en la forma de comunicarse de los estudiantes a través de una interacción positiva, abierta, dialogante, libre de agresiones, lo que ha generado espacios de aprendizaje saludables y constructivos. 
Asimismo, los estudiantes se han valorado como personas, han mostrado una mayor consideración y respeto, así como la capacidad de tolerar otros puntos de vista, y han desarrollado capacidades para reconocer y aprender que un problema puede tener diversas causas y se pueden formular varias alternativas de solución; son más consciente de sus actos en la interacción con sus pares y familiares, y aprecian los conflictos como una ocasión para crecer y desarrollarse, personal y socialmente, aprendiendo nuevas formas de enfrentarse a los problemas.

El programa "Desarrollemos la competencia social para convivir en armonía con los demás" ha generado una mejora significativa: los estudiantes superaron las manifestaciones de conductas de maltrato escolar en sus diferentes manifestaciones, como evidencian los resultados cuantitativos y cualitativos.

Teniendo en cuenta las evidencias tanto cualitativas como cuantitativas, se concluye que el programa de prevención aplicado resultó eficaz para el desarrollo de los aprendizajes propuestos enmarcados dentro de la competencia social. Los estudiantes han integrado habilidades, conocimientos y actitudes para aprender a convivir en el aula, con una mejor disposición para dialogar, expresar sus ideas y sentimientos con asertividad, el saber comprender y ponerse en el lugar del que sufre y del que necesita ayuda, así como ofrecer su amistad y solidaridad en situaciones de maltrato escolar. También ha potenciado las capacidades cognitivas, emocionales, comunicativas e integradoras para afrontar con éxito los conflictos de relación personal e interpersonal que se presentan en la vida diaria y que se asuma un comportamiento positivo y adaptable en la interacción con sus pares, familia u contextos socioculturales.

Por último, consideramos que cualquier intervención educativa dirigida a mejorar la convivencia es inútil si no se considera a las familias, para que juntos enseñemos a convivir mejor con los demás, construyendo de esta manera escuelas y familias saludables en un marco de respeto a la persona. La organización de una actuación educativa rigurosa debe estar orientada a potenciar y desarrollar el aprendizaje de la competencia social con la intencionalidad de prevenir conductas de maltrato escolar. Dicha actuación educativa, más allá de las peculiaridades de programas y de estrategias, debe contemplar actividades que atiendan la compleja constelación estructural de los comportamientos antisociales, es decir, todas las actividades deben promover el desarrollo de habilidades cognitivas, emocionales, comunicativas e integradoras desde una perspectiva de desarrollo ético y moral.

\section{Referencias}

Aguilera, A. Muñoz, G. y Orozco A. (2007). Disciplina, violencia y consumo de sustancias nocivas a la salud en primarias y secundarias de México. Recuperado de http://www.prevenciondelaviolencia.org/system/files/recursos/35 disciplina violencia y consum $\underline{\text { o.pdf }}$

Altarejos, F. (1986). Modelo educativo USAT 2011. Perú: Universidad Católica Santo Toribio de Mogrovejo.

Arismendi, R. (1992). Camino sin retorno. México: Universidad Autónoma del Estado de México.

Chaux, E. (2012). Educación, convivencia y agresión escolar. Colombia: Santillana.

Comisión Nacional para el Desarrollo y Vida sin Drogas. (2007). Il estudio nacional: prevención y consumo de drogas en estudiantes de Secundaria. Recuperado de http://www.opd.gob.pe/cdoc/cdocumentacion/estudio nacional P Escolar 2007 
De Zubirías, F. (2006). Los modelos pedagógicos. Hacia una pedagogía dialogante. Bogotá: Cooperativa Editorial Magisterio.

del Barrio, C., Barrios, A., Meulen, K. y Gutiérrez, H. (2003). Las distintas perspectivas de estudiantes y docentes acerca de la violencia escolar. Estudios de Juventud, 62, 6-79

Defensor del Pueblo-UNICEF (2007). Violencia escolar: el maltrato entre iguales en la ESO 1999-2006 (Nuevo estudio y actualización del Informe 2000). Recuperado de

https://www.defensordelpueblo.es/informe-monografico/violencia-escolar-el-maltrato-entreiquales-en-la-educacion-secundaria-obligatoria-1999-2006-nuevo-estudio-y-actualizacion-delinforme-2000-2007/

Defensor del Pueblo. (2000). Informe sobre la violencia escolar. Madrid: Narcea.

Díaz, M. (2007). Programas de educación para la tolerancia y la prevención de la violencia en los jóvenes. Madrid: Ministerio del Trabajo y Asuntos Sociales.

Fernández, I. (2003). Escuela sin violencia. Resolución de conflictos. México: Narcea.

Frontado, J. (2003). Convivencia familiar basada en valores. México: McGraw Hill.

Galeano, E. (2005). Patas arriba: la escuela del mundo al revés. Madrid: Alianza.

García, V. (1981). Educación personalizada. Madrid: Ediciones Rialp.

Gevaert, J. (1987). El problema del hombre. España: Sígueme.

Gonzales, M. y Negreiros, C. (2001). Curriculum I. Piura: Universidad de Piura.

Hazas, E. (2010). Estrategias de resolución de conflictos en preescolares (Tesis Doctoral). Universidad Complutense de Madrid, España. Recuperado de

http://eprints.ucm.es/10487/1/T31912.pdf

Hernández, R., Fernández, C. y Baptista, P. (2006). Metodología de la Investigación (4a. ed.). México: McGraw-Hill.

Johnson, M. H., Kieling, L. W. y Cooper, S. L. (2013). East coast/west coast art project: a constructivist and technological approach to middle level and higher education collaboration. Art Education, 66(4), 22-27.

Killam, W. K., Roland, C. B. y Weber, B. (2014). Violenceprevention in middle school: a preliminary study. Michigan Journal of Counseling: Research, Theory and Practice, 40(2), 4-11.

Levine, E. y Tamburrino, M. (2014). Bullying among young children: strategies for prevention. Early Childhood Education Journal, 42(4), 271-278.

Martin, A., Del Barrio, C. y Echeita, G. (2003). La intervención para la mejora de la convivencia en los centros educativos: modelos y ámbitos. Infancia y aprendizaje, 26, 79-95.

Mendoza, D. (2012). Bullying. Los múltiples rostros del abuso escolar. Argentina: Brujas.

Oliveros, M. y Barrientos, A. (2007). Incidencia y factores de riesgo de la intimidación (bullying) en un colegio particular de Lima-Perú. Revista Peruana de Pediatría, 60(3), 150-155. 
Oliveros, M., Figueroa, L., Mayorga, G., Cano, G., Quispe, Y. y Barrientos, A. (2009). Intimidación en colegios estatales de secundaria del Perú. Revista Peruana de Pediatría, 62(2), 68-78.

Olweus, D. (1993). Bullying at school: What we know and what we can do. Oxford: Blackwell Publishers.

Ortega, R. y Del Rey, R. (2007). La violencia escolar. Estrategias de prevención. Barcelona: Grao.

Piñero, E. (2010). Características de las relaciones familiares y escolares y roles en la dinámica bullying en estudiantes de Educación Secundaria Obligatoria (Tesis doctoral). Universidad de Murcia, España. Recuperado de https://digitum.um.es/xmlui/handle/10201/17576

Santander, R., Zubarew, G., Santelices, C., Argollo, M., Cerda, L. y Bórquez, P. (2008). Influencia de la familia como factor protector de conductas de riesgo en escolares chilenos. Revista Médica de Chile, 136(3), 317-324.

Segura, M. (2004). Relacionarnos bien. Madrid: Narcea

Segura, M. (2007). Jóvenes y adultos con problemas de conducta. Desarrollo de las competencias sociales. Madrid: Narcea.

Smith, B. H. y Low, S. (2013). The role of social-emotional learning in bullying prevention efforts. Theory into Practice, 52(4), 280-287.

Tójar, J. C. (2006). Investigación cualitativa. Comprender y actuar. Madrid: La Muralla.

Torrego, J. y Moreno, J. (2003). Convivencia y disciplina en la escuela. El aprendizaje de la democracia. Madrid: Alianza.

Trianes, M. y García, A. (2002). Educación socio-afectiva y prevención de conflictos interpersonal en los centros escolares. Revista Interuniversitaria de Formación del Profesorado, 44, 139-174.

Tuvilla, J. (2004). Convivencia escolar y resolución pacífica de conflictos. Sevilla, España: Consejería de Educación y Ciencia.

Valdivieso, P. (2009). Violencia escolar y relaciones intergrupales. Sus prácticas y significados en las escuelas secundarias en la comuna de Peñalolen en Santiago de Chile (Tesis doctoral). Universidad de Granada, España. Recuperado de http://www.ugr.es/ erivera/PaginaDocencia/Posgrado/Documentos/ValdiviesoPablo.pdf

Yepes, J. (2003). Fundamentos de antropología: un ideal de la excelencia humana. Pamplona: Ediciones Eunsa. 